\title{
Pauli blocking and final-state interaction in electron-nucleus quasielastic scattering
}

\author{
Lon-chang (L.C.) Liu ${ }^{\dagger}$ \\ Theoretical Division, Group T-16, Mail Stop B243 \\ Los Alamos National Laboratory, Los Alamos, NM 87545 USA
}

\begin{abstract}
The nucleon final-state interaction in inclusive electron-nucleus quasielastic scattering is studied. Based on the unitarity equation satisfied by the scattering-wave operators, a doorway model is developed to take into account the final-state interaction including the Pauli blocking of nucleon knockout. The model uses only experimental form factors as the input and can be readily applied to light- and medium-mass nuclei. Pauli blocking effects in these latter nuclei are illustrated with the case of the Coulomb interaction. Significant effects are noted for beam energies below $\sim 350 \mathrm{MeV}$ and for low momentum transfers.

$\dagger$ e-mail address: liu@lanl.gov
\end{abstract}

Keywords: Nuclear response function, Pauli blocking.

PACS: 25.30.Dh, 25.30.Fj 


\section{Introduction}

The dominant contribution to electron-nucleus reactions at energies below the pion production threshold comes from quasielastic electron-nucleus scattering[1], in which a target nucleon is knocked out to the continuum by the incoming electron. While exclusive quasielastic experiments can provide detailed nuclear structure information of the struck nucleon, inclusive experiments allow us to study various general properties of the reaction dynamics [2]-[5]. As the final-state interaction (FSI) between the knocked-out nucleon and the residual nucleus can affect the calculated spectra[5], it must be properly evaluated. For exclusive experiments, optical potentials are often used to calculate the FSI [6]-[8]. Because these nonhermitian potentials differ from the potential that binds the nucleon in the nucleus,

they generate nucleon scattering wavefunctions that are not orthogonal to the bound-state wavefunction of the nucleon. This nonorthogonality leads to overestimated (spurious) contribution to nucleon knockout cross sections as the momentum transfer $\vec{q} \rightarrow 0$. Many methods were proposed to restore the orthogonality[9]-[13]. For inclusive experiments, nuclear final states are not measured. Hence, in principle, a real-valued potential is to be used for FSI calculations. If one solves simultaneously the bound-state and scattering problems with a same real-valued potential, then the above-mentioned orthogonality difficulty will not occur. However, very often, particularly in the case of nonrelativistic treatment of FSI in inclusive experiments, one uses phenomenological energy-dependent potentials[14]-[16]. These potentials differ from the potential that binds the nucleon. In this respect, the lack of orthogonality exists in practice and it is of interest to improve the implementation of the required orthogonality in inclusive calculations. In this work, we develop a new approach to FSI in inclusive quasielastic scattering, which does not need an explicit use of potentials while implements the needed orthogonality at all FSI energies on a same footing. 
Because the distortion of the electron waves in the initial and final states can be taken into account by the DWBA method and is of no relevance for the discussion presented in this work, we will, therefore, use plane waves for the electrons so as to show more clearly the effects of blocking spurious knockouts in the new approach. The theory is developed in Section 2 and its application is given in Section 3, Discussion and conclusions are presented in Section 4 .

\section{Electron quasielastic scattering from a nucleus}

The one-photon exchange, one-nucleon knockout amplitude, A, is illustrated in Fig.1 where the four-momenta of the on-shell particles (external lines of the diagram) are denoted by $p_{i}=\left(E_{i}, \vec{p}_{i}\right)$ with $i=(0,1,2, C, A)$. The four-momentum of the photon is $q=p_{0}-$ $p_{2} \equiv(\omega, \vec{q})$. With the Bjorken-Drell convention [17] for the metric, single-particle state normalization, and reaction cross section, the quasielastic scattering differential cross section equals to

$$
\begin{aligned}
\frac{d^{2} \sigma}{d \Omega_{2} d E_{2}}= & \int \frac{(2 \pi)^{4}}{v_{\text {in }}} \sum_{\text {spins }} \delta^{3}\left(\vec{p}_{0}+\vec{p}_{A}-\vec{p}_{1}-\vec{p}_{2}-\vec{p}_{C}\right) \delta\left(E_{0}+E_{A}-E_{1}-E_{2}-E_{C}\right) \\
& \left(\frac{m_{e} M_{A}}{E_{0} E_{A}}\right) \frac{1}{2\left(2 J_{A}+1\right)}|\mathbf{A}|^{2} \frac{\left|\vec{p}_{2}\right| E_{2}}{(2 \pi)^{3}\left(E_{2} / m_{e}\right)} \frac{d \vec{p}_{1}}{(2 \pi)^{3}\left(E_{1} / M_{N}\right)} \frac{d \vec{p}_{C}}{(2 \pi)^{3}\left(E_{C} / M_{C}\right)},(1)
\end{aligned}
$$

where $v_{i n}=E_{0} E_{A} / \sqrt{\left(p_{0} \cdot p_{A}\right)^{2}-p_{0}^{2} p_{A}^{2}}$ is the relative velocity in the initial channel, $J_{A}$ is the spin of the target nucleus, and the summation is over the spin projections of the external particles.

As in any Feynman diagram, the intermediate particles are off-mass-shell particles. This is the case with the intermediate photon, the intermediate nucleon, $j$, and the corresponding residual nucleus, denoted $C(j)$. However, it is useful to put the intermediate heavy nucleus, $C(j)$, on its mass shell and to retain only the positive-energy spinors of the nucleon $j$. This covariant approximation enables one to use the bound-state nuclear wavefunctions given by traditional nuclear structure theories in which the negative-energy component of the wavefunction is not considered. [18] Because the difference among various nuclear masses $M_{C(j)}$ is $\ll M_{N}$, it is also useful to define $M_{C}$ as an average of $M_{C(j)}$ and substitute the former for the latter. One thus has 


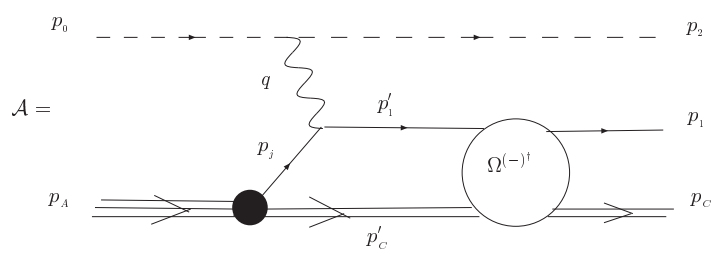

Fig. 1. Amplitude A for quasielastic scattering. The dashed, wavy, solid, and multiple solid lines represent, respectively, the electrons, the photon, the nucleon, and the nuclei. $\Omega^{(-)^{\dagger}}$ is the wave operator for the nucleon final-state interaction. A summation over the target nucleon label $j$ is understood.

$$
\begin{aligned}
\mathbf{A} & =\left(\frac{e e_{p} f\left(q^{2}\right)}{q^{2}}\right) \bar{u}\left(\vec{p}_{2}, s_{2}\right) \gamma_{\nu} u\left(\vec{p}_{0}, s_{0}\right) \sum_{J_{j} \mu_{j}} \sum_{J_{C(j)} s_{C(j)}} \sum_{s_{j} s_{1}^{\prime}} \int \frac{d \vec{p}_{j}}{(2 \pi)^{3}\left(E_{j} / M_{N}\right)\left(E_{C}^{\prime} / M_{C}\right)\left(E_{1}^{\prime} / M_{N}\right)} \\
& \times\left\langle\vec{p}_{1} \frac{1}{2} s_{1} ; \vec{p}_{C} J_{C} s_{C}\left|\Omega_{j}^{(-)^{\dagger}}\right| \vec{p}_{1}^{\prime} \frac{1}{2} s_{1}^{\prime} ; \vec{p}_{C}^{\prime} J_{C(j)} s_{C(j)}\right\rangle\left\langle\vec{p}_{1}^{\prime} \frac{1}{2} s_{1}^{\prime}\left|J^{\nu}(0)\right| \vec{p}_{j} \frac{1}{2} s_{j}\right\rangle \\
& \times\left[\frac{\left\langle\vec{p}_{j} \frac{1}{2} s_{j} ; \vec{p}_{C(j)}^{\prime} J_{C(j)} s_{C(j)}|\Gamma| p_{A} J_{A} s_{A}\right\rangle}{p_{j}^{0}-E_{j}+i \epsilon}\right]
\end{aligned}
$$

In Eq.(2) the abbreviated notations $E_{C}^{\prime} \equiv E_{C}\left(\vec{p}_{C}^{\prime}\right), E_{j} \equiv E_{N}\left(\vec{p}_{j}\right)$ and $E_{1}^{\prime} \equiv E_{N}\left(\vec{p}_{1}^{\prime}\right)$ are used. The square of the four-momentum transfer is $q^{2}=\omega^{2}-|\vec{q}|^{2}$. The four-momentum conservation at each interaction vertex gives $\vec{p}_{1}^{\prime}=\vec{p}_{j}+\vec{q}, p_{1}^{\prime 0}=p_{j}^{0}+\omega, \vec{p}_{j}=\vec{p}_{A}-\vec{p}_{C}^{\prime}$, and $p_{j}^{0}=E_{A}\left(\vec{p}_{A}\right)-E_{C}^{\prime}\left(\vec{p}_{C}^{\prime}\right)$. The $J_{j}, \mu_{j}$ are the total angular momentum and its third component of the $j$-th target proton, and $\sum_{J_{j} \mu_{j}}=Z$ being the total number of the target protons. The $e$ and $e_{p}$ denote, respectively, the electron and proton charges, and the $u(\bar{u})$ and $U(\bar{U})$ the corresponding spinors. The $f\left(q^{2}\right)$ is the $\gamma p p$ form factor and

$$
\left\langle\vec{p}_{1}^{\prime} \frac{1}{2} s_{1}^{\prime}\left|J^{\nu}(0)\right| \vec{p}_{j} \frac{1}{2} s_{j}\right\rangle=\bar{U}\left(\vec{p}_{1}^{\prime}, s_{1}^{\prime}\right) J^{\nu}(q) U\left(\vec{p}_{j}, s_{j}\right)=\int d \vec{x}\left\langle\vec{p}_{1}^{\prime} \frac{1}{2} s_{1}^{\prime}\left|e^{i \vec{q} \cdot \vec{x}} J^{\nu}(\vec{x})\right| \vec{p}_{j} \frac{1}{2} s_{j}\right\rangle
$$

where $J=\left(J^{0}, \vec{J}\right)$ is the electromagnetic current operator. For single-nucleon processes one can represent the target nucleus as an active nucleon $i$ and a corresponding spectator residual nucleus $C(i)$, i.e.,

$$
\left|\vec{p}_{A} J_{A} s_{A}\right\rangle=\sum_{J_{i} J_{C(i)}} F\left(J_{i} J_{C(i)} ; J_{A}\right) \sum_{s_{C(i)} \mu_{i}} C\left(J_{i} \mu_{i}, J_{C(i)} s_{C(i)} \mid J_{A} s_{A}\right)\left|\vec{p}_{i} ; J_{i} \mu_{i}\right\rangle\left|\left(\vec{p}_{A}-\vec{p}_{i}\right) ; J_{C(i)} s_{C(i)}\right\rangle ;
$$




$$
\left|J_{i} \mu_{i}\right\rangle=\sum_{m_{i}, s_{i}} C\left(\frac{1}{2} s_{i}, \ell_{i} m_{i} \mid J_{i} \mu_{i}\right)\left|\frac{1}{2} s_{i}\right\rangle\left|\Phi_{J_{i} \ell_{i} m_{i}}\right\rangle .
$$

Here $\left.F\left(J_{i} J_{C(i)} ; J_{A}\right) \equiv\left[J_{i}^{\nu-1}\left(J_{C(i)}\right) J_{i} J_{A} \mid\right\} J_{i}^{\nu} J_{A}\right]$ is the coefficient of fractional parentage, with $\nu$ being the number of protons in the shell having the momentum $J_{i}$. The $C$ 's are the Clebsch-Gordan coefficients. Upon using the bound-state equation $G_{0} \Gamma \Phi_{b d}=\Phi_{b d}$ (with $G_{0}=\left(p_{j}^{0}-E_{j}+i \epsilon\right)^{-1}$ and $\left.\Phi_{b d}=\left|J_{j} \mu_{j}\right\rangle\right)$, one obtains the covariant single-particle nuclear wavefunction given by

$$
\begin{aligned}
\Phi_{\{j\}}\left(\vec{\lambda}_{j}\right) & \equiv \frac{\left\langle\vec{p}_{j} \frac{1}{2} s_{j} ; \vec{p}_{C(j)}^{\prime} J_{C(j)} s_{C(j)}|\Gamma| \vec{p}_{A} j_{A} s_{A}\right\rangle}{p_{j}^{0}-E_{j}+i \epsilon} \\
& =F\left(J_{i} J_{C(i)} ; J_{A}\right) C\left(J_{j} \mu_{j}, J_{C(j)} s_{C(j)} \mid J_{A} s_{A}\right) C\left(\frac{1}{2} s_{j}, \ell_{j} m_{j} \mid J_{j} \mu_{j}\right) \Phi_{J_{j} \ell_{j} m_{j}}\left(\vec{\lambda}_{j}\right) .
\end{aligned}
$$

Here, $\{j\}$ stands for the ensemble of quantum numbers $J_{j}, \mu_{j}, J_{C(j)}, s_{C(j)}, s_{j}, \ell_{j}, m_{j}$. Furthermore, $\vec{\lambda}_{j}=\eta \vec{p}_{j}-\vec{p}_{C(j)}^{\prime} / A=\vec{p}_{j}-\vec{p}_{A} / A$ with $\eta=(A-1) / A$ is the relative momentum between nucleon $\mathrm{j}$ and the corresponding residual nucleus $C(j)$.

In Eqs.(11)-(44), the states |\rangle and \langle| are covariantly normalized, namely, $\left\langle\vec{k}^{\prime}, s^{\prime} \mid \vec{k}, s\right\rangle=$ $(E(\vec{k}) / M)^{1 / 2} \delta\left(\vec{k}^{\prime}-\vec{k}^{\prime}\right) \delta_{s^{\prime} s}$. On the other hand, in nonrelativistic nuclear theories the states, which we denote |\rangle$\rangle$ and $\left\langle\langle|\right.$, have the normalization $\left\langle\left\langle\vec{k}^{\prime}, s^{\prime} \mid \vec{k}, s\right\rangle\right\rangle=\delta\left(\overrightarrow{k^{\prime}}-\vec{k}\right) \delta_{s^{\prime} s}$. Hence, $|\vec{k}\rangle=|\vec{k}\rangle\rangle(E(\vec{k}) / M)^{1 / 2}$. It follows that $\Phi$ is related to its noncovariantly normalized counterpart, $\phi$, by

$$
\Phi_{J_{j} \ell_{j} m_{j}}\left(\vec{\lambda}_{j}\right)=\left(\frac{E_{j} E_{C(j)} E_{A}}{M_{N} M_{C} M_{A}}\right)^{1 / 2} \phi_{J_{j} \ell_{j} m_{j}}\left(\vec{\lambda}_{j}\right)
$$

where $\phi_{J_{j} \ell_{j} m_{j}}\left(\vec{\lambda}_{j}\right)=R_{j_{j} \ell_{j}}\left(\left|\vec{\lambda}_{j}\right|\right) Y_{m_{j}}^{\ell_{j}}\left(\hat{\lambda_{j}}\right)$. Being dependent on the relative momentum $\vec{\lambda}_{j}$, $\phi$ is a spectral wave function. Its relation to the corresponding shell-model wave function is given in Ref.[19]. Upon introducing Eqs.(2)-(5) into Eq.(11), one can write Eq.(1) in the following compact form:

$$
\frac{d^{2} \sigma}{d \Omega_{2} d E_{2}}=\left(\frac{d \sigma_{M}}{d \Omega_{2}}\right)\left(\frac{m_{e}^{2}}{E_{0} E_{2}} \frac{L_{\mu \nu} W^{\mu \nu}}{\cos ^{2}\left(\theta_{2} / 2\right)}\right)
$$

where $L_{\mu \nu}=\frac{1}{2} \sum_{s_{0} s_{2}}\left[\bar{u}\left(\vec{p}_{0}, s_{0}\right) \gamma_{\mu} u\left(\vec{p}_{2}, s_{2}\right) \bar{u}\left(\vec{p}_{2}, s_{2}\right) \gamma_{\nu} u\left(\vec{p}_{0}, s_{0}\right)\right]$ and

$$
W^{\mu \nu}=\int \frac{(2 \pi)^{3}}{v_{i n}} \delta^{3}\left(\vec{p}_{0}+\vec{p}_{A}-\vec{p}_{1}-\vec{p}_{2}-\vec{p}_{C}\right) \delta\left(E_{0}+E_{A}-E_{1}-E_{2}-E_{C}\right) \frac{\left|\vec{p}_{2}\right| M_{A}}{E_{2} E_{A}}\left|f\left(q^{2}\right)\right|^{2}
$$




$$
\begin{aligned}
& \sum_{\{j\},\{i\}} \int \frac{d \vec{p}_{j} d \vec{p}_{i}}{(2 \pi)^{6}\left(E_{j} / M_{N}\right)\left(E_{i} / M_{N}\right)}\left(\frac{M_{C}}{E_{C(j)}^{\prime}}\right)\left(\frac{M_{C}}{E_{C(i)}^{\prime \prime}}\right)\left(\frac{M_{N}{ }^{2}}{E_{N}\left(\vec{p}_{j}+\vec{q}\right) E_{N}\left(\vec{p}_{i}+\vec{q}\right)}\right) \\
& \frac{1}{2} \sum_{s_{1}^{\prime} s_{1}^{\prime \prime}}\left\langle\vec{p}_{i} \frac{1}{2} s_{i}\left|J^{\mu}(0)\right|\left(\vec{p}_{i}+\vec{q}\right) \frac{1}{2} s_{1}^{\prime \prime}\right\rangle\left\langle\left(\vec{p}_{j}+\vec{q}\right) \frac{1}{2} s_{1}^{\prime}\left|J^{\nu}(0)\right| \vec{p}_{j} \frac{1}{2} s_{j}\right\rangle \Phi_{\{i\}}^{*}\left(\vec{\lambda}_{i}\right) \Phi_{\{j\}}\left(\vec{\lambda}_{j}\right) \\
& \left\langle\left(\vec{p}_{i}+\vec{q}\right) \frac{1}{2} s_{1}^{\prime \prime} ; \vec{p}_{C}^{\prime \prime} J_{C(i)} s_{C(i)}\left|\Omega_{i}^{(-)} I(1, C) \Omega_{j}^{(-)^{\dagger}}\right|\left(\vec{p}_{j}+\vec{q}\right) \frac{1}{2} s_{1}^{\prime} ; \vec{p}_{C}^{\prime} J_{C(j)} s_{C(j)}\right\rangle .
\end{aligned}
$$

The $d \sigma_{M} / d \Omega_{2}$ is the Mott differential cross section and is given by

$$
\frac{d \sigma_{M}}{d \Omega_{2}}=\frac{e^{2} e_{p}^{2}}{(2 \pi)^{2}} \frac{E_{2}^{2}}{\left(q^{2}\right)^{2}} \cos ^{2}\left(\theta_{2} / 2\right) .
$$

In Eq.(8)

$$
I(1, C) \equiv \sum_{s_{1} J_{C} s_{C}} \int\left|\vec{p}_{1} \frac{1}{2} s_{1} ; \vec{p}_{C} J_{C} s_{C}\right\rangle\left\langle\vec{p}_{1} \frac{1}{2} s_{1} ; \vec{p}_{C} J_{C} s_{C}\right| \frac{d \vec{p}_{1} d \vec{p}_{C}}{(2 \pi)^{6}\left(E_{1} / M_{N}\right)\left(E_{C} / M_{C}\right)}=1,
$$

as a result of the completeness of free two-particle states. Consequently,

$$
\Omega_{i}^{(-)} I(1, C) \Omega_{j}^{(-)^{\dagger}}=\Omega_{i}^{(-)} \Omega_{j}^{(-)^{\dagger}} \delta_{i j}
$$

The appearance of $\delta_{i j}$ is a consequence of one-step reaction process in which the residual nucleus acts as a spectator. Because the nucleon $j$ and the residual nucleus can form bound states, the unitary equation of the wave operators is [20]

$$
\Omega_{i}^{(-)} \Omega_{j}^{(-)^{\dagger}} \delta_{i j}=\left(\mathbf{1}-\Gamma_{j}\right) \delta_{i j}
$$

with

$$
\Gamma_{j}=\sum_{n=0}^{n_{\max }}\left|n_{\{j\}}\right\rangle\left\langle n_{\{j\}}\right| \equiv \sum_{n} \Gamma_{j}^{(n)}
$$

Here, $\Gamma_{j}^{(n)}$ denotes the projector to the bound state $\left|n_{\{j\}}\right\rangle$, with $n=0$ denoting the nuclear ground state and $n \neq 0$ the nucleon-emission-stable (NES) excited nuclear states. In the single-step reaction model, $\left|n_{\{j\}}\right\rangle=\left|J_{j}^{(n)}\right\rangle \otimes\left|J_{C(j)}\right\rangle$. Here, a nucleon $j$ is lifted from its ground-state orbital (denoted $J_{j}$ ) to an excited orbital ( denoted $J_{j}^{(n)}, n \neq 0$ ).

The projectors $\Gamma_{j}^{(n)}$ have the properties $\Gamma_{j}^{(n)}=\Gamma_{j}^{(n)^{\dagger}}$ and $\Gamma_{j}^{(n)} \Gamma_{j}^{(m)^{\dagger}}=\Gamma_{j}^{(n)} \delta_{n m}$. These properties allow us to rewrite Eqs.(12) and (13) as

$$
\Omega_{i}^{(-)} \Omega_{j}^{(-)^{\dagger}} \delta_{i j}=\left(\mathbf{1}-\Gamma_{j}\right) \delta_{i j}=\left(\mathbf{1}-\sum_{n=0}^{n_{\max }}\left|n_{\{j\}}\right\rangle\left\langle n_{\{j\}}|\mathbf{1}| n_{\{j\}}\right\rangle\left\langle n_{\{j\}}\right|\right) \delta_{i j} .
$$


This last equation defines the doorway model of the final-state nucleon-nucleus interaction.

Using Eqs.(11)-(14) for the last line of Eq.(8), one obtains, after some angularmomentum recoupling algebra, that

$$
\begin{aligned}
& W^{\mu \nu}=\int \frac{d \vec{p}_{1} d \vec{p}_{C}}{(2 \pi)^{3} v_{i n}} \delta^{3}\left(\vec{p}_{0}+\vec{p}_{A}-\vec{p}_{1}-\vec{p}_{2}-\vec{p}_{C}\right) \delta\left(E_{0}+E_{A}-E_{1}-E_{2}-E_{C}\right) \frac{\left|\vec{p}_{2}\right|}{E_{2}}\left|f\left(q^{2}\right)\right|^{2} \\
& \times\left(\Xi_{I}^{\mu \nu}-\Xi_{I I}^{\mu \nu}\right)
\end{aligned}
$$

with

$$
\Xi_{I}^{\mu \nu}=\frac{1}{2} \sum_{s_{1}} \sum_{J_{j} \mu_{j} \ell_{j} m_{j} s_{j}}\left\langle\left\langle\vec{p}_{j} \frac{1}{2} s_{j}\left|J^{\mu}(0)\right| \vec{p}_{1} \frac{1}{2} s_{1}\right\rangle\right\rangle\left\langle\left\langle\vec{p}_{1} \frac{1}{2} s_{1}\left|J^{\nu}(0)\right| \vec{p}_{j} \frac{1}{2} s_{j}\right\rangle\right\rangle\left|\phi_{\{j\}}\left(\vec{\lambda}_{j}\right)\right|^{2},
$$

and

$$
\begin{aligned}
\Xi_{I I}^{\mu \nu} & =\sum_{n=0}^{n_{\max }} \frac{1}{2} \sum_{s^{\prime} s^{\prime \prime}} \sum_{J_{j} \mu_{j} \ell_{j} m_{j} s_{j}}\left|\phi_{\{j\}}^{(n)}(\vec{\lambda})\right|^{2} \\
& \times\left[\int \frac{d \vec{p}_{j}}{(2 \pi)^{3}} \phi_{\{j\}}^{*}\left(\vec{\lambda}_{j}\right)\left\langle\left\langle\vec{p}_{j} \frac{1}{2} s_{j}\left|J^{\mu}(0)\right|\left(\vec{p}_{j}+\vec{q}\right) \frac{1}{2} s^{\prime \prime}\right\rangle\right\rangle \phi_{\{j\}}^{(n)}\left(\vec{\lambda}_{j}+\eta \vec{q}\right)\right] \\
& \times\left[\int \frac{d \vec{p}_{i}}{(2 \pi)^{3}} \phi_{\{j\}}^{(n) *}\left(\vec{\lambda}_{i}+\eta \vec{q}\right)\left\langle\left\langle\left(\vec{p}_{i}+\vec{q}\right) \frac{1}{2} s^{\prime}\left|J^{\nu}(0)\right| \vec{p}_{i} \frac{1}{2} s_{j}\right\rangle\right\rangle \phi_{\{j\}}\left(\vec{\lambda}_{i}\right)\right],
\end{aligned}
$$

where $\vec{\lambda}=\eta \vec{p}_{1}-A^{-1} \vec{p}_{C}$ is the relative momentum of the nucleon-residual nucleus system in the final state. The momentum conservation at the $\gamma p p$ vertex gives $\vec{\lambda}=\vec{\lambda}_{j}+\eta \vec{q}$. For succinctness of notation, Eqs.(16) and (17) are expressed in terms of noncovariantly normalized nuclear wave functions $\phi_{\{j\}}$, and noncovariant states $\langle\langle|$ and $\left.\mid\rangle\right\rangle$. Consequently, various normalization factors, of the form $(E / M)$, are implicit.

Eq.(15) is illustrated in Fig,2, Its physics content is as follows. The $\Xi_{I}$ leads to cross sections obtained with using plane waves in the final state. The $\Xi_{I I}$ gives the cross sections for the struck nucleon to remain bound. The subtraction of $\Xi_{I I}$ from $\Xi_{I}$ corrects the spurious contribution arising from using plane waves. As we shall see, at $\vec{q}=0$ the subtraction is total; in other words, the spurious proton knockout is completely blocked. Using the well-known 


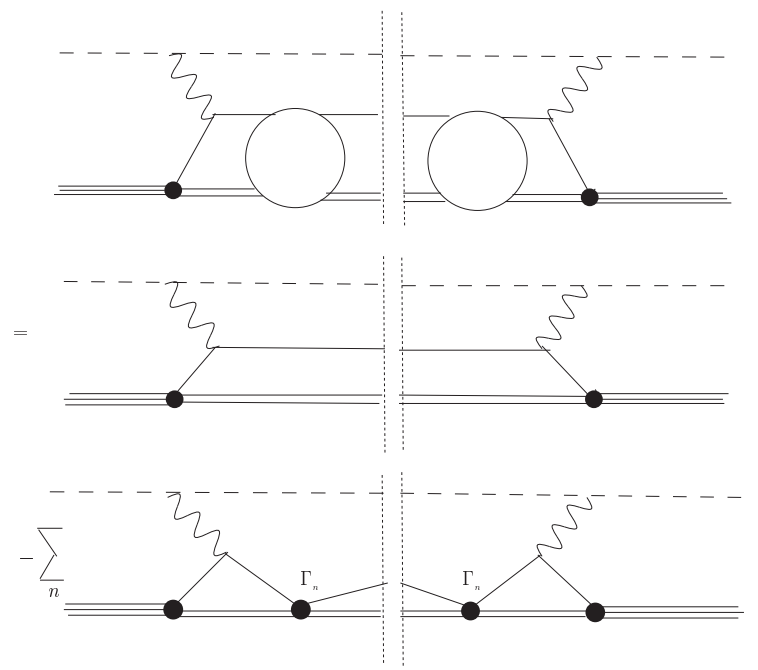

Fig. 2. The doorway model for Pauli-blocking corrections. As in Fig, the subscript $j$ and a summation over it on both side of the graphic equation is implied.

Lorentz-invariant parametrization[21, 22] of the response tensor $W^{\mu \nu}$, one obtains

$$
\frac{d^{2} \sigma}{d \Omega_{2} d E_{2}}=\frac{d \sigma_{M}}{d \Omega_{2}}\left[\left(\frac{\left(q^{2}\right)^{2}}{|\vec{q}|^{4}}\right) R_{L}(\omega,|\vec{q}|)+\left(\frac{q^{2}}{2|\vec{q}|^{2}}-\tan ^{2}\left(\theta_{2} / 2\right)\right) R_{T}(\omega,|\vec{q}|)\right]
$$

in the laboratory frame. Here, $R_{T}$ and $R_{L}$ are, respectively, the transverse and longitudinal response functions with $R_{T}=\sum_{\lambda= \pm 1}\left(\vec{e}_{\vec{q}, \lambda}^{\dagger}\right)_{i} W^{i j}\left(\vec{e}_{\vec{q}, \lambda}\right)_{j}(i, j=1,2,3)$ and $R_{L}=W^{00}=$ $\Xi_{I}^{00}-\Xi_{I I}^{00}$.

\section{Effects of Pauli Blocking}

To illustrate the blocking of spurious nucleon knockout in the doorway model, let us consider the Coulomb scattering only. In this latter case, $J^{\mu}=(\hat{\rho}, \overrightarrow{0})$. Hence, $R_{T}=0$ and

$$
\frac{d^{2} \sigma}{d \Omega_{2} d E_{2}}=\frac{d \sigma_{M}}{d \Omega_{2}} \frac{\left(q^{2}\right)^{2}}{|\vec{q}|^{4}} R_{L}
$$

In the second quantization $\hat{\rho}(\vec{x})=\hat{\psi}^{\dagger}(\vec{x}) \hat{\psi}(\vec{x})$. Upon using the nonrelativistic two-component proton field $\hat{\psi}(\vec{x})=(2 \pi)^{-3 / 2} \int d \vec{k} \sum_{\xi} e^{i \vec{k} \cdot \vec{x}} a_{\vec{k}, \xi} \chi_{\xi}$, one finds that the two matrix elements of $J^{0}$ in the first square brackets in $\Xi_{I}^{00}$ equal to $\delta_{s_{1} s_{j}}$ while the two matrix elements of $J^{0}$ in 
$\Xi_{I I}^{00}$ become, respectively, $\delta_{s^{\prime \prime} s_{j}}$ and $\delta_{s^{\prime} s_{j}}$. Consequently,

$$
R_{L}=\int \frac{d \vec{K}}{v_{i n}} \delta^{3}\left(\vec{q}+\vec{p}_{A}-\vec{K}\right) \frac{\left|\vec{p}_{2}\right|}{E_{2}}\left|f\left(q^{2}\right)\right|^{2} R(\omega,|\vec{q}|)
$$

with

$$
\begin{gathered}
R(\omega,|\vec{q}|)=\int \frac{d \vec{\lambda}}{(2 \pi)^{3}} \delta\left(\omega+E_{A}-E_{1}-E_{C}\right) \\
{\left[\sum_{j}\left(\left|\phi_{\{j\}}\left(\overrightarrow{\lambda_{j}}\right)\right|^{2}-\left|\phi_{\{j\}}(\vec{\lambda})\right|^{2}\left|F_{\{j\}}^{00}(\vec{q})\right|^{2}\right)-\sum_{j}{ }^{\prime} \sum_{n \neq 0}\left|\phi_{\{j\}}^{(n)}(\vec{\lambda})\right|^{2}\left|F_{\{j\}}^{0 n}(\vec{q})\right|^{2}\right] .}
\end{gathered}
$$

In obtaining Eqs.(201) and (21) we used the relations $d \vec{p}_{1} d \vec{p}_{C}=d \vec{K} d \vec{\lambda}$ (with $\vec{K} \equiv \vec{p}_{1}+\vec{p}_{C}$ ) and

$$
\int \frac{d \vec{p}_{i}}{(2 \pi)^{3}} \phi_{\{j\}}^{(n) *}\left(\vec{\lambda}_{i}+\eta \vec{q}\right) \phi_{\{j\}}\left(\vec{\lambda}_{i}\right)=\int d \vec{r}_{i} e^{i \vec{q} \cdot \vec{r}_{i}} \psi_{\{j\}}^{(n) *}\left(\vec{r}_{i}\right) \psi_{\{j\}}\left(\vec{r}_{i}\right)=F_{\{j\}}^{0 n}(\vec{q})
$$

The $\Sigma^{\prime}$ in Eq.(21) indicates that not every target proton is involved in a $0 \rightarrow n$ transition. Hence, $\sum_{j}^{\prime} 1 \equiv Z^{\prime} \leq Z$. The $\delta$ function in Eq.(21) constrains the energy loss $\omega$ and makes $\omega$ depend on $\vec{\lambda}^{2}$ and the average proton separation energy $B=M_{1}+M_{C}-M_{A}$.

In Eq. (21),$F_{\{j\}}^{00}(\vec{q}) \equiv F_{\{j\}}^{\text {g.s. } \rightarrow \text { g.s. }}(\vec{q})$ is the nuclear (ground-state) form factor of the $j$-th proton with the property $F_{\{j\}}^{00}(0)=1$. For $n \neq 0, F_{\{j\}}^{0 n}(\vec{q}) \equiv F_{\{j\}}^{g . s . \rightarrow n}(\vec{q})$ are the transition form factors, and $F_{\{j\}}^{0 n}(0)=0$. Consequently, when $\vec{q} \rightarrow 0, R \rightarrow 0$; i.e., the knockout of a target proton is completely blocked at $\vec{q}=0$. We have noted that experimental form factors are not parametrized with respect to an individual proton but rather with respect to the whole nucleus as a function of $|\vec{q}|$. (Henceforth, $|\vec{q}|$ is denoted as $q$ for a succinct notation.) It is, therefore, appropriate to introduce

$$
\begin{aligned}
& F_{\{j\}}^{00}(\vec{q})=\frac{1}{Z} F_{A}^{00}(q) \equiv F^{00}(q), \\
& F_{\{j\}}^{0 n}(\vec{q})=\frac{1}{Z^{\prime}} F_{A}^{0 n}(q) \equiv F^{0 n}(q) \quad(n \neq 0) .
\end{aligned}
$$

The $q$-dependence of PBC can be obtained by integrating over all energy loss in Eq.(21). Using the completeness relation

$$
\int \frac{d \vec{\lambda}}{(2 \pi)^{3}}\left|\phi_{\{j\}}\left(\vec{\lambda}_{j}\right)\right|^{2}=\int \frac{d \vec{\lambda}_{j}}{(2 \pi)^{3}}\left|\phi_{\{j\}}\left(\vec{\lambda}_{j}\right)\right|^{2}=1
$$


one obtains

$$
\int d \omega R(\omega, q)=Z\left(1-\left|F^{00}(q)\right|^{2}-\beta \sum_{n \neq 0}^{n_{\max }}\left|F^{0 n}(q)\right|^{2}\right) \equiv Z L(q) .
$$

The ratio $\beta \equiv Z^{\prime} / Z$ depends on nuclear excitation mechanisms. The function $L(q)$ gives the probability for a struck proton to leave the nucleus. Eq.(25) shows how the doorway and Fermi gas models differ. In the Fermi gas model, the nucleon density distribution $\left|\psi\left(\vec{p}_{j}\right)\right|^{2}$, is assumed to be $\theta\left(\left|\vec{p}_{j}\right|-k_{F}\right)$ where $k_{F}$ is the Fermi momentum. Because of the Pauli principle, this box-type momentum-space density distribution blocks $\psi\left(\vec{p}_{j}\right) \rightarrow \psi\left(\vec{p}_{j}+\vec{q}\right)$ transitions whenever $\left|\vec{p}_{j}+\vec{q}\right| \leq k_{F}$. For realistic density distributions, there is no such sharp momentum cutoff in transitions. Instead, the $\psi\left(\vec{p}_{j}\right)$ to $\psi^{(n)}\left(\vec{p}_{j}+\vec{q}\right)$ transition can occur at any given $\vec{q}$ with the probability $\left|F^{0 n}(q)\right|^{2}$. Hence, $\left|F^{00}(q)\right|^{2}+\beta \sum_{n \neq 0}\left|F^{0 n}(q)\right|^{2}$ is the probability that the struck nucleon remains bound. With a minus sign in front of this last quantity, the second and third terms in Eq.(25) give the blocking correction to nucleon knockout in a realistic nucleus. We name this correction the Pauli-blocking correction (PBC) because it is a consequence of the Pauli exclusion principle.

A comment on Eq.(25) is in order. While form factors $F^{00}$ have been determined experimentally for a large number of nuclei, experimental information on transition form factors $F^{0 n}(n \neq 0)$ is much less systematic. However, in nuclei with mass number $A \leq 5$ there is no NES excited states. Consequently, only the term $\left|F^{00}\right|^{2}$ is needed in Eq.(25). The $L(q)$ can, therefore, be calculated exactly for these light nuclei with the use of experimental form factors.

In Fig, 3 , the functions $L(q)=1-\left|F^{00}(q)\right|^{2}$ for two light nuclei are shown. In both cases $L(q)=0$ at $q=0$ and $L(q) \rightarrow 1$ when $q>2.7 \mathrm{fm}^{-1}$. Graphically, the PBC is represented by $1-L(q)$ which is the vertical distance between the curve and the horizontal line passing through $L(q)=1$. Fig. 3 shows the PBC is complete (i.e., 100\%) at $\mathrm{q}=0$ and how it decreases with increasing $q$. Since there is only one bound state in ${ }^{3} \mathrm{He}$ and ${ }^{4} \mathrm{He}$ (the ground states), $1-\left|F^{00}(q)\right|^{2}$ represents an exact calculation of $L(q)$ for these nuclei. In nuclei with mass number $A \geq 6$, there are NES states and its number increases with $A$. To illustrate the effects of NES states in $1 p$-shell nuclei, we show in Fig 4 the function $L(q)$ of ${ }^{12} \mathrm{C}$, assuming $\beta=1$ in Eq.(25) . The PBC effects due to $\left|F^{00}\right|^{2}$ and $\left(\left|F^{00}\right|^{2}+\left|F^{0,2^{+}}\right|^{2}\right)$ are given, respectively, 


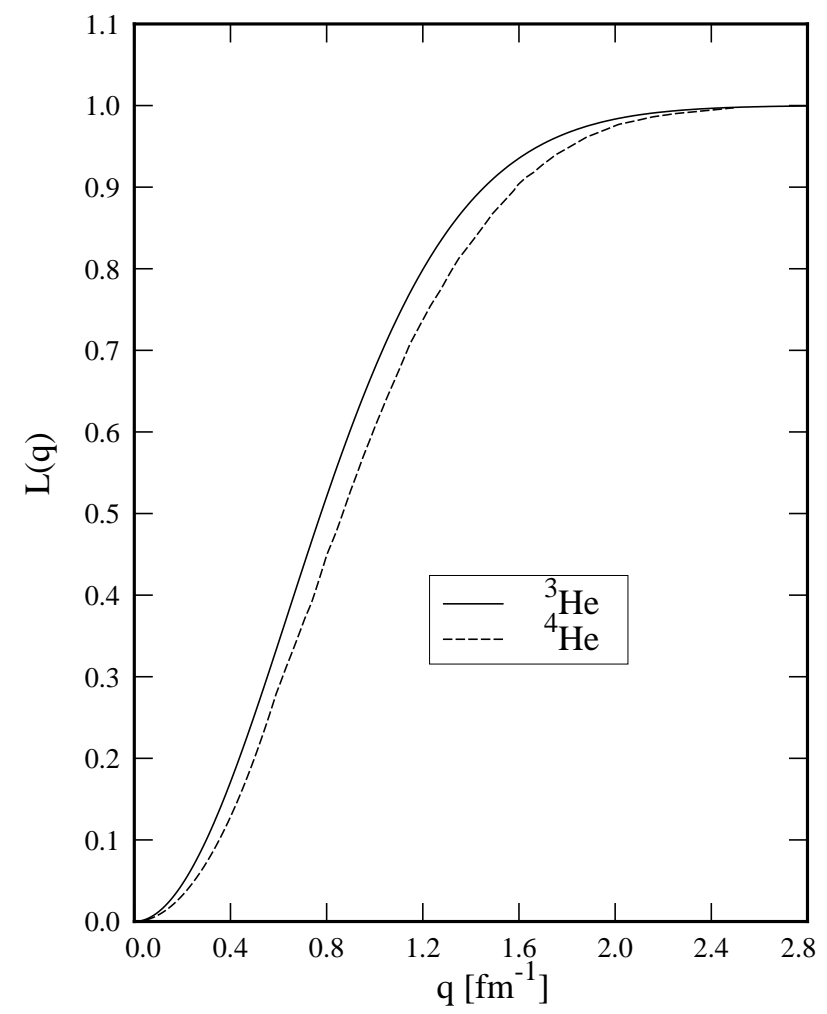

Fig. 3. Function $L(q)=1-\left|F^{00}(q)\right|^{2}$ for nuclei ${ }^{3} \mathrm{He}$ and ${ }^{4} \mathrm{He}$.

by the dashed and solid curves in the figure. Here $2^{+}$is the $4.44 \mathrm{MeV}(T=0)$ excited state. The dot-dashed curve further includes the PBC arising from transitions to the NES states [23]-28] at $7.12 \mathrm{MeV}\left(1^{-}, T=0\right), 9.64 \mathrm{MeV}\left(3^{-}, T=0\right)$, and $14.1 \mathrm{MeV}\left(4^{+}, T=0\right)$. Since the proton separation energy in ${ }^{12} \mathrm{C}$ is $15.11 \mathrm{MeV}$, the inclusion of these four states should take into account most of the NES transition strength. As one can see from Fig, 4, the most important effects of $\left|F^{0 n}\right|^{2}(n \neq 0)$ comes from the transition to the first $2^{+}$excited state at $4.44 \mathrm{MeV}$. The inclusion of other three states brings in only small additional effects. One could expect that, in general, only a limited number of transitions to NES states needs to be considered in medium-mass nuclei. The relative importance of PBC effects due to different doorway channels can be evaluated from comparing the corresponding $\int L(q) d q$. We have found that $\int\left(1-\left|F^{00}(q)\right|^{2}\right) d q$ (integration of the dashed curve) differs from $\int\left(1-\left|F^{00}(q)\right|^{2}-\right.$ $\left.\sum_{n}\left|F^{0 n}(q)\right|^{2}\right) d q,\left(n=2^{+}, 1^{-}, 3^{-}, 4^{+}\right)$(integration of the dot-dashed curve) by less than $2 \%$. 


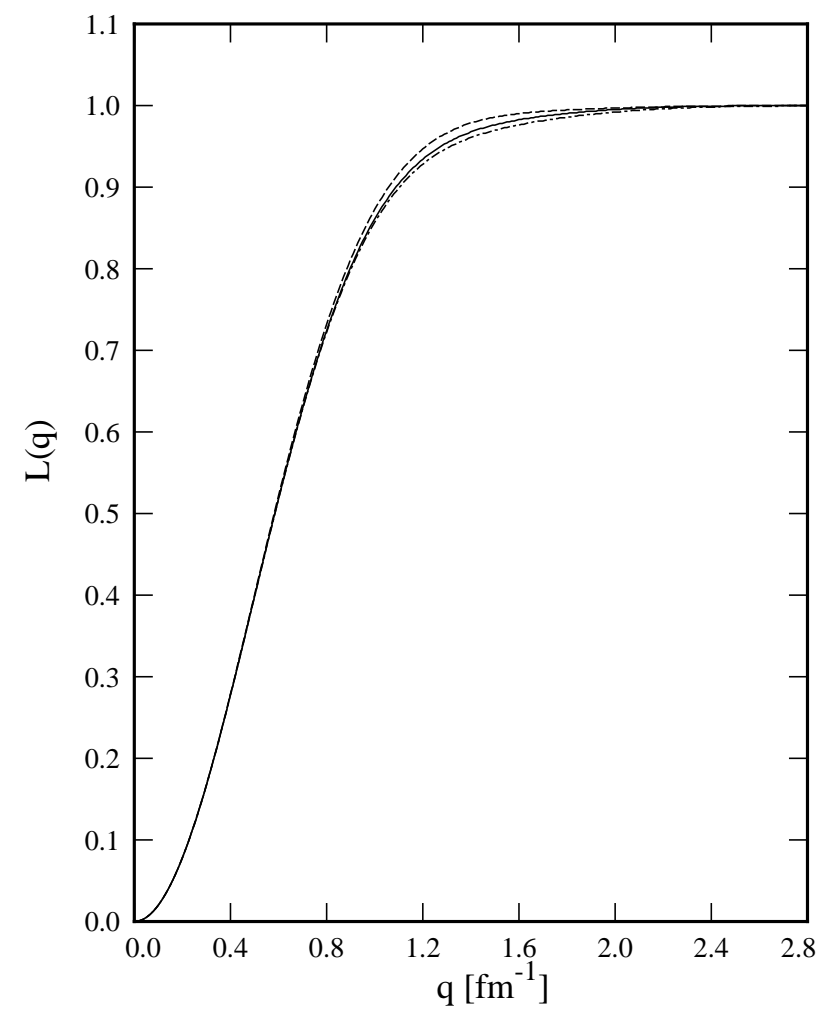

Fig. 4. Functions $L(q)$ for ${ }^{12} C$. Dashed curve: $L=1-\left|F^{00}\right|^{2}$. Solid curve: $L=1-\left|F^{00}\right|^{2}-\left|F^{0,2+}\right|^{2}$. Dot-dashed curve: $L=1-\left|F^{00}\right|^{2}-\sum_{n}\left|F^{0 n}\right|^{2}\left(n=2^{+}, 1^{-}, 3^{-}, 4^{+}\right)$.

In the following calculations of $\mathrm{PBC}$ in ${ }^{12} \mathrm{C}$, we will, therefore, use the term $\left|F^{00}\right|^{2}$ only.

In Fig.5, we show PBC effects on inclusive cross sections of quasielastic scattering from ${ }^{3} \mathrm{He}$ and ${ }^{12} \mathrm{C}$ at $E_{0}=200 \mathrm{MeV}$ and $\theta_{2}=60^{\circ}$ as a function of the energy loss $\omega$. For ${ }^{12} \mathrm{C}$, realistic separation energies $B_{p}=15$ and $B_{s}=35 \mathrm{MeV}$ were used respectively for the $1 p-$ and $1 s$-shell protons. These shell-dependent separation energies give rise to the shoulder in the ${ }^{12} \mathrm{C}$ spectra. As we can see, the PBC is significant in both nuclei. To quantify the integrated PBC effects on the cross section, let us define

$$
\delta=\frac{\left(d \sigma / d \Omega_{2}\right)^{n o P B C}-\left(d \sigma / d \Omega_{2}\right)^{P B C}}{\left(d \sigma / d \Omega_{2}\right)^{n o P B C}} .
$$

The values of $\delta$ in ${ }^{3} \mathrm{He}$ and ${ }^{12} \mathrm{C}$ are given in Table 1 where a blank entry represents a $\delta<1 \%$. As one can see from the table, the PBC decreases with increasing energy and scattering angle 


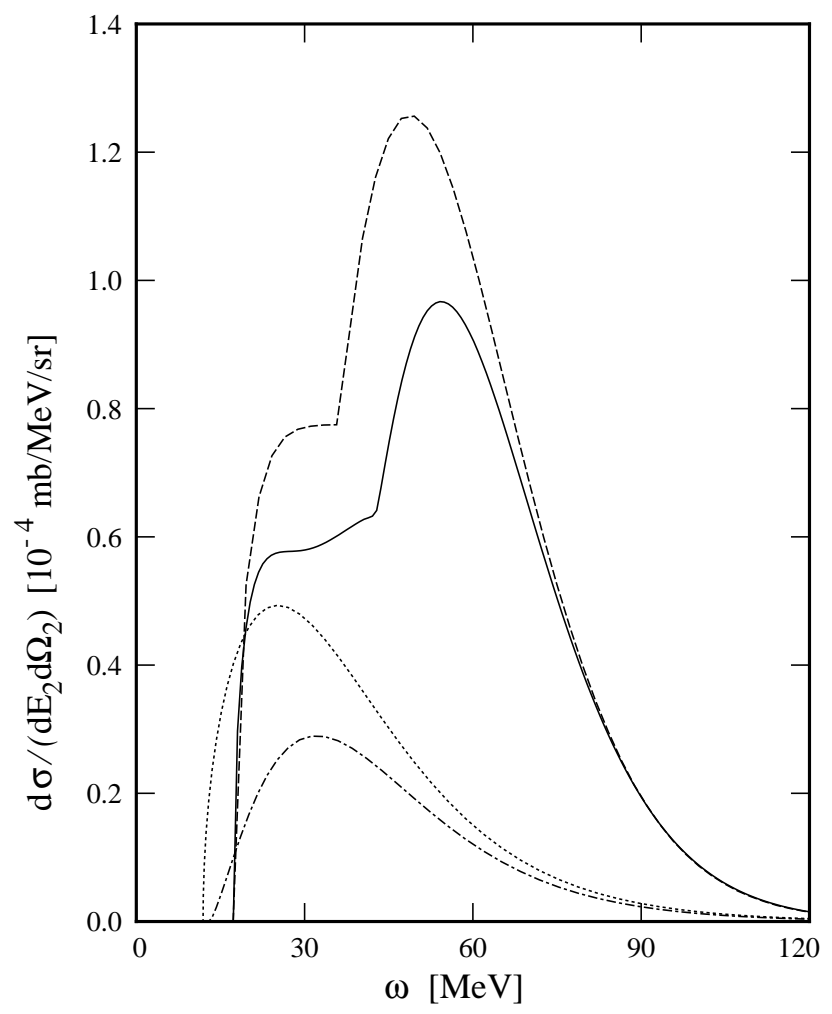

Fig. 5. Inclusive lab. cross sections $d \sigma /\left(d E_{2} d \Omega_{2}\right)$ at $E_{0}=200 \mathrm{MeV}$ and $\theta_{2}=60^{\circ}$ as a function of energy loss $\omega$. Dotted curve: ${ }^{3} \mathrm{He}$ without PBC. Dot-dashed curve: ${ }^{3} \mathrm{He}$ with PBC. Dashed cureve: ${ }^{12} \mathrm{C}$ without PBC. Solid curve: ${ }^{12} \mathrm{C}$ with $\mathrm{PBC}$.

Table 1

Pauli blocking correction $\delta[\%]$.

\begin{tabular}{|c||c|c|c||cc|c|c|}
\hline $\mathrm{E}_{0}[\mathrm{MeV}]$ & ${ }^{3} \mathrm{He}:$ & $\theta_{2}=30^{\circ}$ & $\theta_{2}=45^{\circ}$ & $\theta_{2}=60^{\circ}$ & ${ }^{12} \mathrm{C}: \quad \theta_{2}=30^{\circ}$ & $\theta_{2}=45^{\circ}$ & $\theta_{2}=60^{\circ}$ \\
\hline 200 & 78 & 58 & 40 & 63 & 37 & 18 \\
\hline 350 & 44 & 18 & 6 & 21 & 3 & \\
\hline 500 & 18 & 3 & & 3 & & \\
\hline
\end{tabular}

and becomes negligible at $E_{0}=500 \mathrm{MeV}$ and $\theta_{2}=60^{\circ}$. Indeed, we have noted that under this latter experimental condition the momentum transfers $|\vec{q}|$ contributing to the bulk of the cross sections are greater than 2 and $2.4 \mathrm{fm}^{-1}$ in ${ }^{3} \mathrm{He}$ and ${ }^{12} \mathrm{C}$, respectively. These large 
$q$ lead to negligible PBC (see Figs $[3$ and 4 ).

The $R_{L}$ of ${ }^{3} \mathrm{He}$ and ${ }^{12} \mathrm{C}$ have been measured at $|\vec{q}|=300 \mathrm{MeV} / \mathrm{c}[29]$, [30]. Since at $\mathrm{q}=300 \mathrm{MeV} / \mathrm{c}$ the effect of $\mathrm{PBC}$ in ${ }^{12} \mathrm{C}$ is unimportant (see Fig,4), we compare, therefore, in Fig, the longitudinal response functions of ${ }^{3} \mathrm{He}$ given by the doorway model at $|\vec{q}|=300$ $\mathrm{MeV} / \mathrm{c}$ with the data[29]. As one can see, the $\mathrm{PBC}$ is very important at small $\omega$ 's. The inclusion of PBC improves the position of the peak of the calculated spectrum.

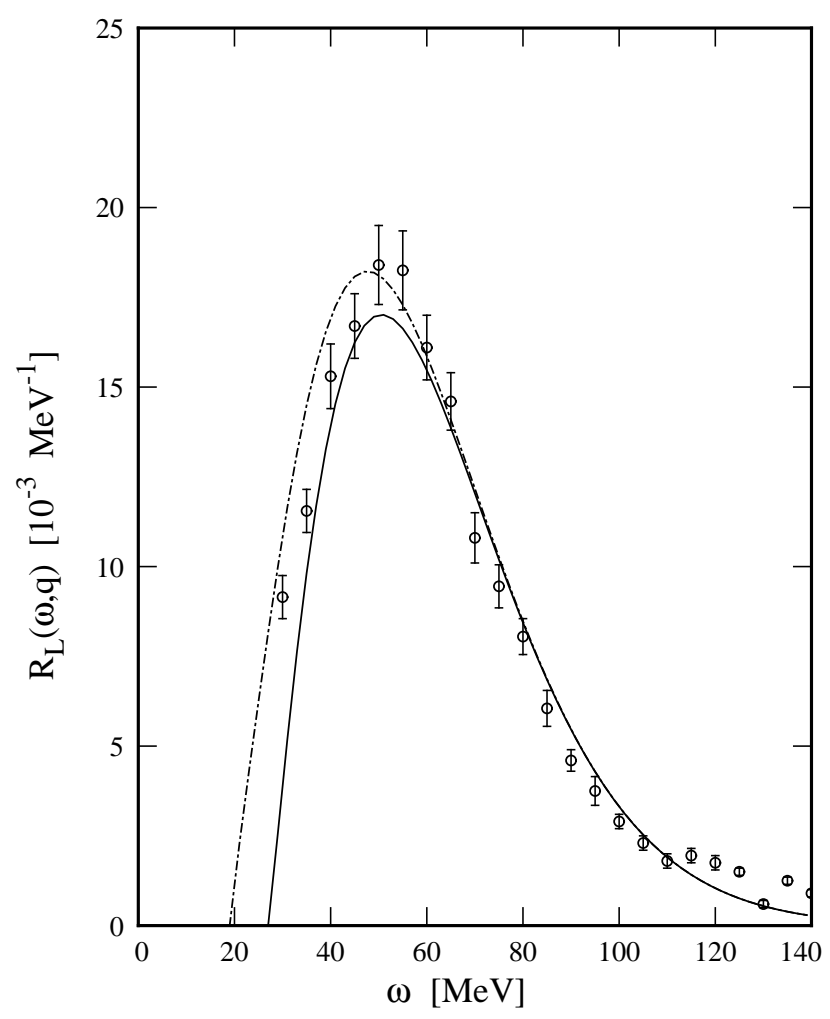

Fig. 6. Longitudinal response functions of ${ }^{3} \mathrm{He}$ at $|\vec{q}|=300 \mathrm{MeV} / \mathrm{c}$. Dot-dashed curve: without PBC. Solid curve: with PBC. Data are from Ref.29.

\section{Discussion and Conclusions}

By using the unitarity equation of the wave operators, we have developed a doorway model for the nucleon final-state interaction in inclusive quasielastic scattering. The model does not rely on the use of potentials; the inputs to the calculation are the experimentally determined form factors. For nuclei with mass numbers $A \leq 5$, the doorway calculation is 
exact. For $1 p$-shell nuclei such as ${ }^{12} \mathrm{C}$ the model can be calculated to a very good approximation with only using the measured ground-state (g.s.) nuclear form factor. One could expect that this latter approximation equally holds for $1 d$ - and $1 f$-shell nuelci. At the present time the application of the doorway model to heavy-mass nuclei is hindered by the lack of a systematic experimental knowledge of the NES transition form factors in these nuclei. It is worth finding out whether the use of a few important experimentally known NES form factors would suffice. Further studies are called for. Our study shows that the Pauli blocking of spurious nucleon knockout is important when the electron energies are below $350 \mathrm{MeV}$ (Table 1). The PBC is also important when momentum transfers are small. The doorway approach derived in this work represents a useful alternate to the various FSI approaches proposed in the literature. It is calculationally simple and can be easily applied to the study of inclusive quasielastic scattering from light and medium-mass nuclei.

\section{References}

[1] Herbert Uberall, Electron Scattering from Complex Nuclei, Part B, Academic Press, New York and London (1971), pp.703-747.

[2] L.S. Celenza, W.S. Pong, M.M. Rahman, and C.M. Shakin, Phys. Rev. C 26 (1982) 320.

[3] H. Meier-Hajduk, U. Oelfke, and P.U. Sauer, Nucl. Phys. A 499637 (1989) .

[4] Omar Benhar, Donal Day, and Ingo Sick, Rev. Mod. Phys.80, 189 (2008).

[5] K.S. Kim and L.E. Wright, Phys. Rev. C76, 044613 (2007).

[6] K. Nakamura and N.Izutsu, Nucl. Phys. A259, 301 (1976).

[7] J. Mougey, M. Bernheim, A. Bussièere, A. Gillebert, Phan Xuan Hô, M. Pirou, D. Royer, I. Sick, and G.J. Wagner, Nucl. Phys. A262, 461 (1976).

[8] C.R. Chinn, A. Picklesimer, and J.W. Van Orden, Phys. Rev. C 40, 1159 (1989).

[9] Louis S. Celenza and C.M. Shakin, Phys. Rev. C 20, 385 (1979).

[10] Q. Haider and J.T. Londergan, Phys. Rev. C 23 (1981) 19.

[11] S. Boffi, F. Cannata, F. Capuzzi, C. Giusti, and F.D. Pacati, Nucl. Phys A379, 509 (1982). 
[12] J.I. Johansson, H.S. Sherif, and F. Ghoddoussi, Nucl. Phys. A 665, 403 (2000).

[13] F. Capuzzi, C. Giusti, F.D. Pacati, and D.N. Kadrev, Ann. Phys.(N.Y.) 317, 492 (2005).

[14] Y. Horikawa, F. Lenz, and Nimai C. Mukhopadhyay, Phys. Rev. C22 1680 (1980).

[15] T. de Forest, Jr., Nucl. Phys. A132 305 (1969).

[16] Y. Kawazoe, G. Takeda, and Matsuzaki, Prog. Theor. Phys. 541394 (1975).

[17] J.D. Bjorken and S.D. Drell, Relativistic Quantum Mechanics, McGraw-Hill Book Co., New York (1964).

[18] L. Celenza, L.C. Liu, and C.M. Shakin, Phys. Rev. C 11 (1975) 1593, appendix B.

[19] L.C. Liu and C.M. Shakin, Nuovo Cim. 53A. 142 (1979).

[20] Leonard S. Rodberg and R.M. Thaler, Introduction to the Quantum Theory of Scattering, Academic Press, New York and London, 1967, p.182.

[21] S.D. Drell and J.D. Walecka, Ann. Phys. (N.Y.) 2818 (1964).

[22] John Dirk Walecka, Electron Scattering for Nuclear and Nucleon Structure, Cambridge University Press, 2001.

[23] J.B. Flanz, R.S. Hicks, R.A. Lindgren, G.A. Peterson, A. hotta, B. Parker, and R.C. York, Phys. Rev. Lett.41, 1642 (1978).

[24] Y. Torizuka, M. Oyamada, K. Nakahara, K. Sugiyama, Y. Kojima, T. Terasawa, K. Itoh, A. Yamaguchi, and M. Kimura, Phys. Rev. Lett.22, 544 (1969).

[25] A. Nakada, Y. Torizuka, and Y. Horikawa, Phys. Rev. Lett. 27745 (1971), errata: 27, 1102 (1971).

[26] R.M. Haybron, M.B. Johnson, and R.J. Metzger, Phys.Rev. 156, 1136 (1967).

[27] Hall Crannel, Phys. Rev.148, 1107 (1966).

[28] I. Ahmad, J. Phys. G3, 1327 (1977).

[29] K. Dow, S. Dytman, D. Beck, A. Bernstein et al., Phys. Rev. Lett. 61, 1706 (1988).

[30] P. Barreau, M. Bernheim, J. Duclos, J.M. Finn, et al., Nucl. Phys. A402 515 (1983). 